\title{
Transient flow reversal combined with sustained embolic prevention in transcervical revascularization of symptomatic and highly-emboligenic carotid stenoses for optimized endovascular lumen reconstruction and improved peri- and post-procedural outcomes
}

\author{
Mariusz Trystuła ${ }^{1}$, Piotr Musiałek ${ }^{2}$ \\ ${ }^{1}$ Department of Vascular Surgery, John Paul II Hospital, Krakow, Poland \\ ${ }^{2}$ Department of Cardiac and Vascular Diseases, Jagiellonian University, John Paul II Hospital, Krakow, Poland
}

Adv Interv Cardiol 2020; 16, 4 (62): 495-506
DOI: https://doi.org/10.5114/aic.2020.102134

\section{Introduction}

Several fundamental advancements have been developed both in embolic protection systems and in technical approaches to reduce the perioperative stroke rates associated with carotid stenting [1-6]. Those developments have been recently reinforced by the progress in carotid stent design [7] that today allows to effectively sequestrate the atherosclerotic plaque, minimizing intra-procedural and preventing post-procedural embolism (second-generation carotid stents, mesh-covered stents) [8]. Transcervical carotid artery stenting under transient flow reversal is a surgical technique that entered its clinical use nearly 2 decades ago [3-5]. Recently, the technique has gained a new momentum with an accumulating data-based realization of its two fundamental advantages: (1) robust cerebral protection by flow reversal is operational against embolism even prior to any endovascular approach to the lesion (i.e., 'protected' lesion crossing), is continuous, and is highly effective throughout the procedure, and (2) avoiding cerebral embolism associated with aortic arch cannulation [3-5, 9]. Accumulating results from transcarotid artery revascularization (TCAR, using the EnRoute flow reversal system, Silk Road) studies show low stroke/death rates comparable to carotid endarterectomy while maintaining the minimally invasive benefits of carotid stenting $[10,11]$. The transcervical approach, which circumvents a number of embolic-risk maneuvers inherent to transfemoral carotid stenting, and with its highly effective proximal cerebral protection [9], has a strong potential to become, in surgical hands at least (as the system requires surgical, rather than percutaneous, exposure of the common carotid artery, CCA), the preferred method of carotid artery stenting (CAS) in the near future. Paired with the progress in carotid stent technology [7, 12], it may well challenge carotid endarterectomy (CEA) as the carotid artery revascularization method of choice not only in standard-risk lesions, but also in symptomatic and highly emboligenic lesions [11].

Our experience, over the years, involved a high-proportion proximal cerebral protection use [6, 13-15] and it also involved our introduction [16] and every-day clinical practice all-comer patient-and-lesion evaluation [15-17] of the MicroNET-covered embolic prevention stent. As an International 'TCAR Center of Excellence' (2018) we systematically paired the TCAR system (EnRoute, Silk Road) with our routine use of the CGuard embolic prevention stent system (InspireMD) to minimize not only intra-procedural but also post-procedural cerebral embolism in relation to the index carotid lesion $[8,12]$. This strategy combines, on the one hand, optimized intra-procedural cerebral protection with carotid plaque sequestration, optimized endovascular lumen reconstruction, and sustained embolic prevention on the other [3-5, 9, 12]. We have found the TCAR system not only clinically effective and operator-friendly but also naturally compatible with the MicroNET-covered stent and hence developed a concept of 'combining the best (in carotid neuroprotection technology) with the best (in carotid stent technology)' and steadily increased our TCAR use in high-risk and very high-risk patients and lesions. Unfortunately, the

Corresponding author:

Piotr Musialek MD, PhD, Department of Cardiac and Vascular Diseases, Jagiellonian University, John Paul II Hospital, 80 Pradnicka St, 31-202 Krakow, Poland, fax: +48 1261433 32, e-mail: pmusialek@szpitaljp2.krakow.pl

Received: 30.11.2020, accepted: 20.12.2020. 
SilkRoad TCAR system has become unavailable to us with the COVID-19 pandemic flight and transportation restrictions, prompting us to explore ways to answer a thus unmet, important clinical need.

\section{Aim}

The present technical note, with its imaging and clinical correlates, describes how to (1) achieve an effective proximal cerebral protection through a transcervical access transient flow reversal kit based on consumables routine in vascular surgery and interventional vascular medicine, and (2) optimize its use by pairing it with the MicroNET-covered stent system for an effective plaque sequestration with endovascular lumen reconstruction and sustained embolic prevention.

\section{Material and methods}

In a series of consecutive patients in whom transfemoral access for CAS was not feasible for anatomic reasons while a minimally-invasive transcarotid intervention was deemed preferable over CEA (NeuroVascular Team decision-making), we performed transcervical CAS with the MicroNET-covered stent system (CGuard) under a robust transient flow reversal that was accomplished by employing vascular surgery and interventional vascular medicine routine materials and devices (Figure $1 \mathrm{~A}$ ). The MicroNET-covered stent system has been characterized in detail previously $[7,15,16]$ (see summarized information provided in Figure 1 legend). CGuard bench testing demonstrated its excellent adaptability (SmartFit feature) and radial force similar to that of the Precise stent (Cordis) [7]. CGuard clinical and angiographic corelab evaluation [15] confirmed its optimal adaptability to the local anatomy, with an optimal technical, angiographic and clinical performance across the whole spectrum of all-comer patients and lesions (from the thrombus-containing to highly-calcific $[15,17])$ and indicated an absence of stent-related thrombosis and a low restenosis rate [17-20]. An important feature of the CGuard system is the MicroNET coverage of any potential residual plaque prolapse between the nitinol frame struts, making it 'protected' (neutralized, non-embolizing) [21].

Diffusion-weighted magnetic resonance imaging (DW-MRI) was performed within 14 days before (baseline) and within 10 days after the procedure (control). Results were evaluated for consistency.

This work was approved by the local Ethics Committee, and all patients provided informed consent.

\section{Results}

Study patients presented with a recent left (dominant) hemispheric stroke (Patient 1 and Patient 4) or recent left (dominant) hemispheric crescendo transient ischemic attacks (TIAs, Patient 1 and Patient 4). They were aged 63-76 years; women/men ratio was $1: 1$. Three patients had Leriche syndrome (Patient 2, Patient 3, and Patient 4) whereas Patient 1 had a two-vessel percutaneous coronary intervention for concomitant critical coronary artery disease and a failed transfemoral access for CAS on the day before the study index procedure. The study patients had severe clinical heart failure (echocardiographic left ventricular ejection fraction of 20-35\%) with prior episodes of hospitalization-requiring exacerbations (recently stable on maximized medical treatment) and/or severe chronic obstructive pulmonary disease (3/4 and $1 / 2$ of the study group, respectively). Two had a short neck, and one had a prior symptomatic (stroke) occlusion of the contralateral carotid artery (Patient 1, cf. Figure 2 B). Prior to the procedure, the operators became familiar with individual anatomy specificities (detailed pre-procedural duplex ultrasound; in addition 2 patients presented with CT angiography performed in the referring hospital). Consistent with our routine strategy of 'local-first' anesthesia in transcervical CAS, all procedures were performed under local anesthesia. There was no clinical or technical need to convert to general anesthesia.

The patients were on aspirin (75 mg o.d.), high-dose statin (atorvastatin $80 \mathrm{mg}$ o.d. or rosuvastatin $40 \mathrm{mg}$ o.d.), and an angiotensin-converting enzyme inhibitor and a $\beta$-blocker. Three patients were on a second antiplatelet agent prior to the index procedure (clopidogrel or prasugrel) whereas in one, the second antiplatelet agent (clopidogrel $600 \mathrm{mg}$ ) was administered immediately after the procedure. Anti-hypertensive medications were withheld on the morning of the index procedure day to increase the arterial blood pressure (the procedural 'baseline' arterial blood pressure of optimally $160-180 \mathrm{~mm} \mathrm{Hg}$ if spontaneous) and thus the likelihood to enhance collateral flow and, if feasible, increase the 'back' pressure [22] that constitutes the principle drive of the flow-reversal neuroprotection $[9,13,14,23,24]$. Atropine (0.5-1 mg i.v.) was given routinely prior to lesion predilatation while unfractionated heparin was titrated to maintain the activated clotting time (ACT) of at least $250 \mathrm{~s}[9,14,19]$ throughout the intervention.

The transcervical CAS procedure in this study all performed within one month. Routinely available materials to assemble flow reversal kits are shown in Figure $1 \mathrm{~A}$. The CCA (surgical, and as proximal as feasible to facilitate the lesion no-touch technique) access is presented in Figure 1. Fundamental steps of surgery were according to the literature [3-5] and incorporated our TCAR system experience. An immediate post-procedural access site closure result is exemplified in Figure 1 B. In 2 patients (Patient 1 and Patient 4) endovascular CCA clamping was performed using the FlowGate (Stryker) or MoMa (Medtronic) balloon catheter. Because the role of external carotid artery (ECA) clamping in trans-cervical flow reversal is debated $[3-5,23,24]$, in our transcervical MoMa procedure we used the two-balloon version of the catheter (ECA and CCA). However, the ECA balloon had 
Figure 1. A
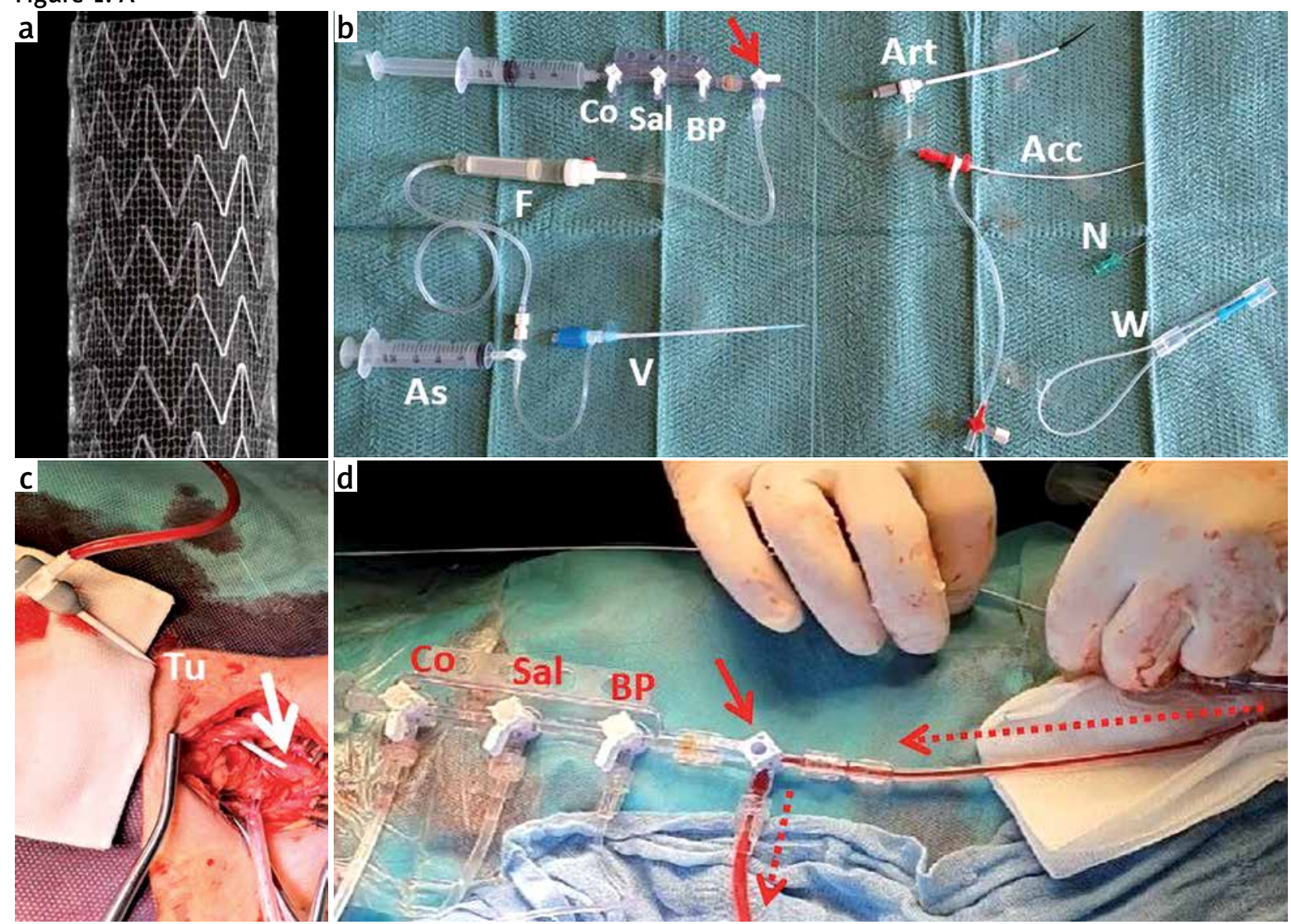

Figure 1. Technical aspects of carotid artery lumen reconstruction using the transcervical route under transient flow reversal, with the MicroNET-covered stent use for plaque sequestration and sustained embolic prevention. A - The armamentarium, vascular access, and principles of proximal cerebral protection. $\mathbf{a}-$ The MicroNET-covered carotid stent consisting of a widely open-cell nitinol frame covered, on the outside, with a MicroNET sleeve. The aperture size of the mesh pores is 150-180 $\mu \mathrm{m}$, similar to the size of pores in filters used in filter-protected CAS. The open-cell stent frame strut thickness is $0.24 \mathrm{~mm}$ and the nitinol frame open-cell area is $21.66 \mathrm{~mm}^{2}$ (i.e., the largest among open-cell carotid stents). The closed cell, proprietary MicroNET is made of a single-knitted PET fiber of $20 \mu \mathrm{m}$ in thickness, forming mesh cells of only $0.023-0.032 \mathrm{~mm}^{2}$, i.e., the most dense closed-cell area among contemporary stents. The system is available in a typical range of lengths (20 to $60 \mathrm{~mm}$ ) and diameters $(6$ to $10 \mathrm{~mm})$. b - Flow reversal armamentarium: The initial arterial access sheath ('Acc', 4F or 5F), the working sheath ('Art', 8F or 9F) with the needle ('N') and a wire ('W'), flow reversal operation central stopcock with a handle (red arrow), 3-port manifold (blood pressure port, 'BP', $0.9 \%$ heparinized saline port, 'Sal', and a contrast port, 'Co'), the filter ('F'; here - a blood transfusion filter with a modified inflow spike tip to enable harboring potentially large embolic particles) connected at the outflow end to the venous sheath (' $V$ '). An additional $20 \mathrm{ml}$ aspiration syringe ('As') may be connected either at the venous sheath side tubing stopcock (such a placement of 'As' prevents inadvertent re-aspiration of the filter-captured debris to the syringe and their erroneous passage back to the arterial line) or directly at the central stopcock (cf. simplified set-up in Figure 1B-j that does not involve intra-arterial blood pressure monitoring and a manifold, but a single-syringe port that can harbor, depending on the procedural step, either a contrast syringe or a saline syringe or an aspiration syringe). Note that we manually pre-shape arterial sheaths to improve coaxial alignment to the CCA lumen and reduce the artery dissection risk. In the case of tunneling (cf. c, 'Tu') that is highly advisable for a maximally co-axial alignment of the working sheath in the case, in particular, of a deep positioning of the common carotid artery and/or a short neck, the standard (short) access needle needs to be replaced with a longer one. c - The common carotid artery (CCA) sheath entry site; display of a deep anatomic positioning of the artery that explains the role of tunneling for a maximally co-axial sheath alignment. This example illustrates why the trans-cervical access is, at least with the current technology, a routinely surgical rather than percuta- 
neous access. Surgical CCA exposure and access allow a controlled closure of the CCA entry site with a suture and direct hemostasis control, allow the access angle improvement; all in all, the risk of inadvertent events such as puncture of the CCA posterior wall or of other vessels in the vicinity and the risk of the artery access site bleeding is minimized (see also Figure $1 \mathrm{~B}-\mathrm{a}$ and $1 \mathrm{~B}-\mathrm{b}$ ). Furthermore, surgical cutdown and CCA exposure is mandatory in the case of performing surgical (rather than endovascular, cf. Patient 1 and Patient 4) clamping of the CCA using a tourniquet (the green arrow in Figure 1B-a and 1B-b; cf. Patient 2 and Patient 3 in Figure 2). Endovascular part of the procedure - insertion of the lesion predilatation balloon, under flow reversal, through the $Y$ connector that is linked either directly to the working sheath side arm tubing (in the case of surgical CCA clamping; $c f$. Patient 1 and Patient 4 in Figure $2 \mathrm{~A}$ ) or to the balloon catheter $Y$ connector (in the case of endovascular clamping; $c f$. Patient 2 and Patient 3 in Figure 2 A). The red arrow illustrates positioning of the central stopcock, with the arterial sheath side tube flow directed exclusively to the femoral vein (example in Figure $1 \mathrm{~B}-\mathrm{d}$ ) through a filter (not shown in Figure $1 \mathrm{~A}-\mathrm{d}$ that is a zoom on the $\mathrm{Y}$ connector visible on the right side of the image, the central stop-cock (red arrow). Dotted arrows indicate flow direction. See Figure $2 \mathrm{~A}$ for corresponding angiographic images. B - Technical aspects of the procedure; (a) and (b) illustrate the importance of tunneling ('Tu') in the case of the deep anatomical location of CCA and/or short neck and/or short CCA with a low positioning of the bifurcation; (c) shows the importance of the sheath manual pre-shaping to maximize alignment with the CCA lumen; (d) illustrates the redundant balloon catheter length when using the balloon catheters of length that enables transfemoral access to CCA (such as FlowGate, Stryker, in (d) or MoMa, Medtronic, in (e)), indicating the need for customization (shortening) that would have the additional beneficial effect of reducing flow resistance; (e) demonstrates the working set-up, with the central 3-way stopcock connected, on the one side, to the MoMa catheter $Y$ connector, on the other to 3-tap manifold (in this setup a $\mathrm{BP}$ line, $0.9 \% \mathrm{NaCl}$ line, and the contrast line), and on the third port - to the filter entry port. Inflating the CCA balloon of the MoMa catheter (inflation syringe, 'Infl') will result in flow cessation, with the 'back' pressure value that can be read on the monitor screen (see (g)). Turning the handle of the central stopcock $90^{\circ}$ clockwise will result a brisk reverse flow driven by the 'back' pressure of, in this case, 55/42 $\mathrm{mm} \mathrm{Hg}$. The saline in the tubing by the filer entry port ('sal') will be spontaneously pushed into the filter by the blood coming from the brain, with the deposit of any plaque embolic debris in the filter. The emboli-filtered blood then spontaneously returns, via the filter exit port, through the tubing to the venous sheath side arm ('Ven' shown in (d)) to the venous circulation of the patient. Principal limiting factors of the flow reversal robustness are the 'back' pressure generated by the cerebral collateral circulation (that is an anatomy-driven factor not modifiable other than increasing the systemic pressure), the diameter of the tubing as well as and any other potential 'bottlenecks' in the circuit, the total length of the access catheters (if used) and the tubing (both should not be longer than needed), and the stenosis severity (until effectively predilated). The latter is not a factor relevant for the operating capacity of the flow reversal protection because any debris mobilized with predilatation will get robustly moved to the filter with increased back flow resulting from relieving the stenosis severity. Dotted red arrows indicate flow direction. (f) and (g) are the ECG (green trace) and invasive blood pressure (red trace) monitoring at baseline (f) and during flow reversal (g). The 'back' pressure of, in this case, 55/42 $\mathrm{mm} \mathrm{Hg}$ is the force that drives reversed flow from the arterial to the venous circulation (in our setup, the femoral vein though other large veins, such as jugular vein may also be used) through a filter-containing temporal (intraprocedural) fistula setup. (h) is the moment of turning the central stop-cock handle $90^{\circ}$ clockwise, resulting in a robust flow from the diseased ICA towards the femoral vein; the reversed flow briskly replaces (i) the saline in the tubing (Sa) with the blood $(\mathrm{Bl})$ that from now on carries to the filter ( $c f$. Figure $2 \mathrm{~B}$ ) any athero-thrombotic embolic debris generated with lesion crossing, predilatation and stent insertion ( $c f$. Figure $2 \mathrm{~A}$ ). ( $\mathrm{j}$ ) is a simplified version of the system, with the arteriovenous flow directly from the sheath side arm tubing, through the central stopcock, to the filter and then to the side arm of the venous sheath. With this simplified setup, there is no 'back' pressure monitoring. Each individual syringe needed at the particular stage of the procedure (i.e., saline syringe, contrast syringe, aspiration syringe) gets connected directly to the central stopcock (red arrow). The point captured in (j) is the moment of transferring the blood from the operated artery (that has just been aspirated to the syringe), through the filter, to the femoral vein. $(\mathbf{k})$ and $(\mathbf{l})$ are typical immediate post-procedural results after surgical closure of the arterial access site, yielding, with the location of the cut, an optimal cosmetic result ('Re' is Redon drain). The skin incision length may be routinely reduced to $\approx 2 \mathrm{~cm}[2]$ according to local anatomic conditions and surgeon's preferences. The long-term cosmetic effect, with the incision site in a natural anatomic line is barely visible, and it is significantly superior to that of conventional CEA; in addition skin sensation in the neck is normal that has a very practical role, for instance, during shaving [34] 

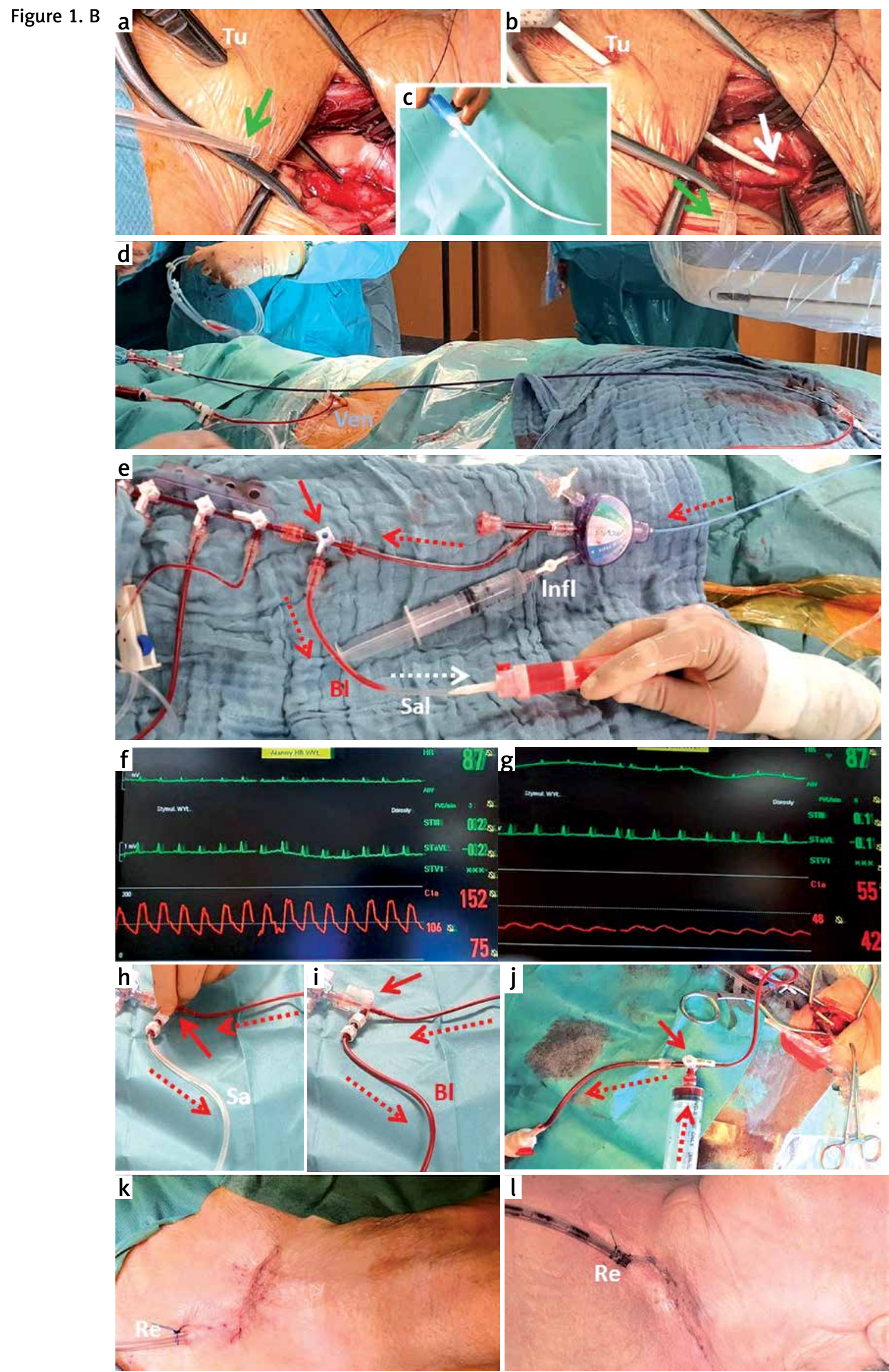
Figure 2. A

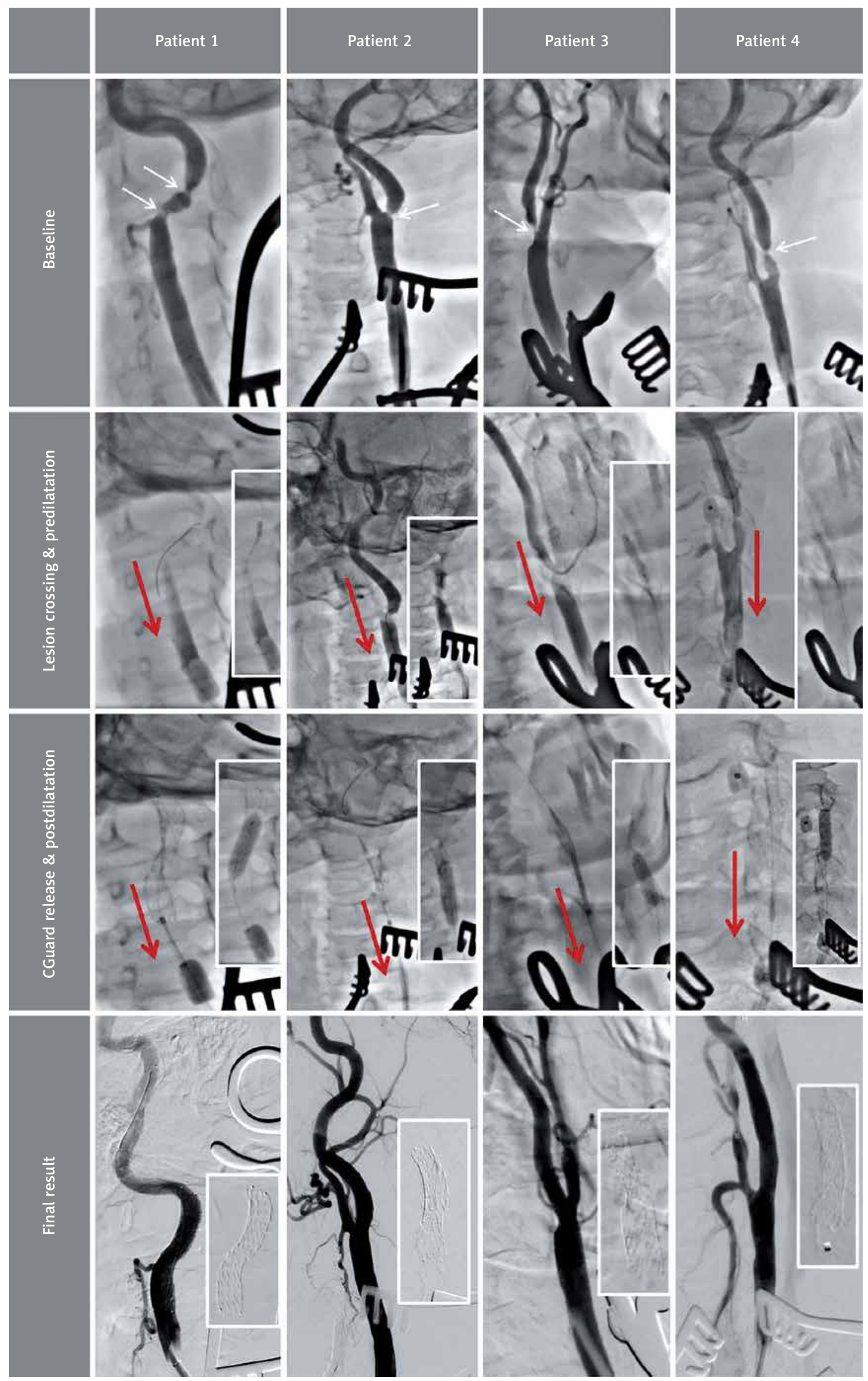


Figure 2. B

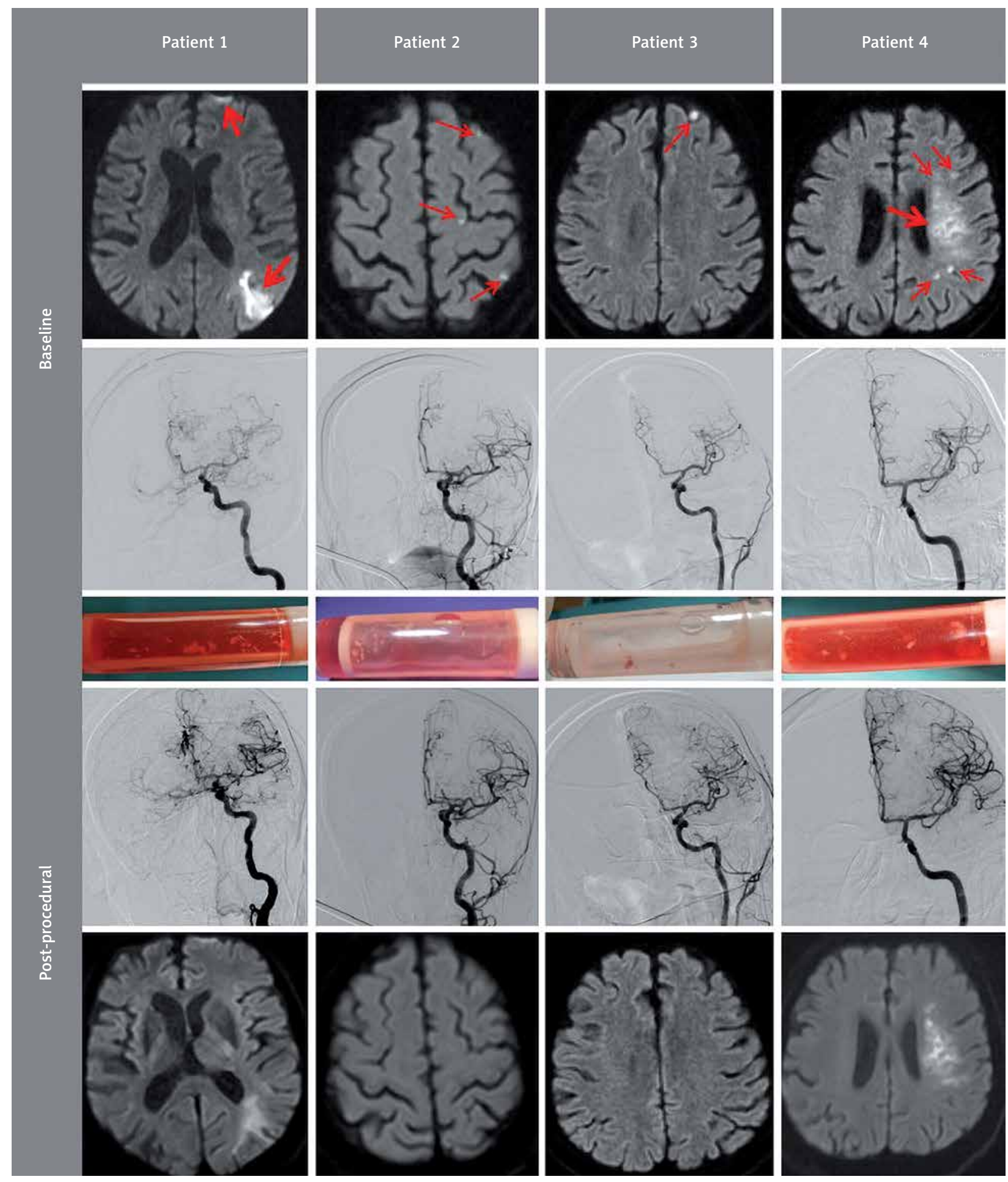


Figure 2. Angiography, cerebral ischemia imaging, and the flow reversal filter material in transcervical carotid artery stenting under flow reversal. A - Angiography at fundamental stages of the procedure. The top panel shows baseline angiographic images after establishing vascular access (cf. Figure 1) in four consecutive patients with sub-acutely symptomatic internal carotid artery stenosis (Duplex images were consistent with a thrombus-containing tight ICA lesion in all subjects, Patient 1 had a tandem stenosis). All patients are left-hemispheric dominant, and the symptom-causing lesion was in the left internal carotid artery (LICA). Two patients (Patient 1 and Patient 4) presented with subacute ( $\leq 14$ days) left-hemispheric stroke (cf. Figure 2 B); two other subjects had crescendo TIAs that were presumed to be hemodynamic due to a very high lesion severity on Duplex imaging (baseline diffusion-weighted imaging provided evidence for concomitant spontaneous plaque embolism, $c f$. Figure 2 B). The second panel from the top illustrates lesion crossing and predilatation under flow reversal (red arrows). CCA clamping was endovascular in Patient 1 and Patient 4 (see inflated CCA balloons in the bottom of the images in Patient 1 and Patient 4, FlowGate and MoMa, respectively) and surgical (tourniquet; $c f$. Figure 1) in Patient 2 and Patient 3. In all cases the guidewire was WhisperMS 0.014'. Predilatation (insets) was performed using coronary balloons of $3.5 \mathrm{~mm}, 3.5 \mathrm{~mm}, 4.0 \mathrm{~mm}$ and $3.0 \mathrm{~mm}$, respectively at 10-12 atm. Backward washout of the contrast upon lesion predilatation (compare images in the third panel with those in the second panel) is consistent with an effective spontaneous transport of any debris to the filters that are shown in Figure 2 B. The second panel from the bottom shows stent placement and implantation under continued flow reversal (CGuard $9.0 \times 40 \mathrm{~mm}$, CGuard $9.0 \times 30 \mathrm{~mm}$, CGuard $10.0 \times 30 \mathrm{~mm}$, and CGuard $9.0 \times 30 \mathrm{~mm}$ respectively), as well as (insets) sequential post-dilatation optimizations of the stents that we perform routinely $(6.5 \mathrm{~mm}$ balloon in Patient $1,6.0 \mathrm{~mm}$ balloon in Patient 2, and $5.5 \mathrm{~mm}$ balloon in Patient 3 and Patient 4). Final angiographic results of the procedures are demonstrated in the bottom panel, consistent with a full, optimal endovascular reconstruction of the lumen of the artery with $0 \%$ residual stenosis in all cases, full patency of the branches, and a full respect of the anatomy (note the SmartFIT characteristics of the stent - self-adjustment of the widely open nitinol frame, with an adequate 'self-tapering' in case of a significant ICA/CCA diameter mismatch - best illustrated in Patient 3, alleviating a need for any 'tapered' versions of the device; for the final stent images see the insets). B - Cerebral imaging at the point of subacute clinical symptoms triggering referrals for revascularization, and the post-procedural controls. The top panel is diffusion-weighted magnetic resonance (DW-MRI) cerebral imaging the point of symptoms that triggered intervention (revascularization was, in all cases, within 14 days), illustrating acute/subacute lesions in all patients. Small embolic foci are depicted with thin arrows whereas thick arrows indicate relatively large ischemic zones that were consistent with clinically significant, lasting stroke symptoms. The second panel from the top shows cerebral angiography prior to the symptomatic carotid artery revascularization (injections directly via the CCA sheath). Patient 1 (a physician) had undergone a symptomatic occlusion of the right internal carotid artery 4 years earlier. The middle panel shows post-procedural photographs of the filters that were part of the flow reversal temporal arteriovenous fistula (shunt) circuit in these patients (cf. Figure 1). Flow reversal, as our default procedure strategy in such patients, taken together with the lesion 'no touch' strategy until flow reversal establishment, enabled a $100 \%$ effective protection of the brain against the plaque material in all subjects (see control cerebral images in the bottom for comparison with the baseline images). Absence of any new post-procedural lesions on DW-MRI images is consistent with an effective capture of all embolic debris in the filters. The fourth panel from the top is post-procedural cerebral angiograms that are consistent with a normalized ipsilateral cerebral supply in all cases (in Patient 1 note also a marked improvement of the collateral supply to the right hemisphere). The bottom panel presents control DW-MRI images that were acquired within 3-10 days after revascularization. In Patient 4, 1.5T scanner was used for baseline DW-MRI imaging whereas the control scan was performed with 3T (the 1.5T scanner was not operational at the point of control imaging). Note that optimal intraprocedural cerebral protection by transient flow reversal, combined with an effective plaque containment by the MicroNET-covered second-generation carotid stent (embolic prevention) resulted in a total absence of peri-procedural cerebral embolism that is an established surrogate for peri- and post-procedural stroke risk in relation to the index lesion [35] 
to be placed distally due to the fixed distance between the CCA and ECA balloon in the MoMa system (which is in contrast to the former Parodi Anti-Emboli System or the Gore Flow Reversal system with the ECA balloon independent of the CCA balloon, allowing individual anatomy-specific adjustment of the CCA and ECA balloon positioning with an increased likelihood of excluding ECA proximal branch(es) [13].

Anatomical considerations and technical aspects of the procedure (including routine off-the-shelf consumables that we used to assemble filter-containing flow reversal shunts between the operated carotid artery lumen and a femoral vein) are presented in Figure 1. Figure 2 shows angiographic documentation of the procedures with the wire, balloon, and stent data provided in the Figure 2 legend. Flow reversal duration was respectively $4 \mathrm{~min} 20 \mathrm{~s}, 5 \mathrm{~min} 10 \mathrm{~s}, 6 \mathrm{~min} 20 \mathrm{~s}$ and $6 \mathrm{~min} 50 \mathrm{~s}$ (5 min $40 \mathrm{~s}$ on average). Index ICA flow reversal was well-tolerated in the study cohort, except for a transient intolerance (in the last $\approx 90 \mathrm{~s}$ of the procedure) in the patient with contralateral ICA occlusion (Patient 1, the procedure was continued normally and the intolerance symptoms resolved upon restitution of antegrade flow). There were no clinical or technical complications, and all cases showed an excellent angiographic result consistent with optimal reconstruction of the diseased vessel (Figure $2 \mathrm{~A}$ ). The results were consistent across the different patients and the different mode of CCA clamping - surgical (via tourniquet, Figure 1) or endovascular (via inflation of the CCA balloon, Figures 12 ). In the patient concluding the present series, the procedure was finalized with intravascular ultrasound (IVUS) control of the lumen reconstruction quality. IVUS demonstrated a full stent expansion and its excellent apposition in absence of any plaque prolapse. The residual area stenosis was negligible (8\%); there was a full, optimal endovascular reconstruction of the diseased vessel lumen.

The filters used in the shunt contained embolic material in all cases (Figure $2 \mathrm{~B}$ ). The embolic material capture was $100 \%$ effective as control DW-MRI scans (Figure 2 B) showed absence of any new embolism. There was a natural evolution of the cerebral index infarcts in Patient 1 and Patient 4 that had caused clinical stroke symptoms prompting urgent referral for carotid revascularization. The small embolic pre-procedural DW-MRI lesions in Patient 2, Patient 3 and Patient 4 were no longer detectable on the control scans (and they were also not visible on control FLAIR imaging). Evaluation of cerebral flow evolution in relation to DW-MRI imaging confirmed a combination of embolic and hemodynamic cerebral injury as mechanisms underlying presenting symptoms [25]. Thus the embolic debris collected in filters (Figure 2 B) confirmed the highly emboligenic characteristics of the carotid plaques in the study patients as indicated by the clinical characteristics (highly symptomatic lesions) and the Duplex and angiographic lesion imaging in relation to the baseline cerebral DW-MRI scans (Figure 2). Neurologic examination at $24-48 \mathrm{~h}$ post-procedurally revealed no new deficits. On day 3 or 4 post-procedurally the patients were discharged home (Patient 1, Patient 2 and Patient 3) or to a stroke rehabilitation centre as pre-arranged by the referring hospital (Patient 4). Discharge Duplex Doppler showed a normal stent lumen and normal in-stent velocities in all cases. Routine post-discharge evaluation was performed in the vascular surgery outpatient clinic at 10-14 days and was normal. Duplex and clinical follow-up, including neurologic examination, are scheduled at 30 days and then yearly. Follow-up DW-MRI examinations are scheduled at 90 days.

\section{Discussion}

The first fundamental finding from this work is that performing transcervical CAS with minimally-invasive surgical access (surgical exposure of the CCA) and transient flow reversal employing routinely available off-theshelf sheaths and/or catheters and other materials (such as stopcocks, manifolds, tubing, and blood-compatible filters) is feasible and is relatively simple and uncomplicated for operators with experience in transcervical access and experience in CAS. Another finding is that this approach, similar to standard TCAR, appears to perform very well technically and clinically with the state-of-theart second-generation carotid stent (MicroNET-covered stent, CGuard). This is important because the use of conventional (first-generation, single-layered) carotid stents in TCAR (that provides an optimal cerebral protection but, nevertheless, the one that is limited to the revascularization procedure) does not protect against post-procedural embolism and strokes [10, 11, 26, 27]. This shortcoming is consistent with the relationship between the first-generation stent plaque prolapse and post-procedural DW-MRI embolism and strokes that are not prevented by a classic single-layer closed-cell design [8, 12, 28-30]. Conversely, the MicroNET-covered stent provides a clinically effective plaque sequestration and vessel healing that is free of plaque prolapse-related cerebral embolism, and an optimal post-procedural embolic prevention in absence of any thrombosis or restenosis excess [17-19, 30, 31]. While larger patient series and longer follow-up periods are needed, accumulating multi-centric data indicate vascular and clinical durability of the CGuard reconstruction of normal carotid anatomy [17-19, 30, 31].

A combination of minimally-invasive (surgical) direct cervical access, optimized intra-procedural cerebral protection by transient flow reversal using a filter-assisted arteriovenous shunt, and sustained post-procedural embolic prevention using a MicroNET-covered stent may well become a new standard of care in symptomatic [1, 8] or increased-risk [32] carotid stenosis patients. This minimally invasive approach, 'combining the best with best' appears at an immediate line to be ideal for pa- 
tients at increased risk for CEA who are not suitable for transfemoral access. This is particularly relevant as the use of large-bore flow clamping/reversal catheters through the transradial route is debatable because of an increased risk of the radial artery damage, its elimination as a potential arterial graft [33], and an increased risk of the radial access patency loss. The strategy described in the present communication may well be applied to other high-risk patient groups [33] and it has the potential to become the patient-preferred strategy over CEA [34]. It needs to be undertood that the neck base is not (with the cuttent technologies at least) the area for any routine percutaneous asscess. This is due to the fundamental risks that - with a fully percutaneous approach - include the risk of CCA dissection with a 'bad' angle in particular (deep CCA location, short neck, examples oin $1 A-c$ and $1 \mathrm{~B}-\mathrm{a} / \mathrm{b})$, the risk of CCA posterior wall puncture, vagal nerve injury, and the neck uncontrollable haematoma with the consequences of CCA occlusion, thrombosis, and embolism.

Transcervical CAS does not require a long arteriotomy, avoids the course of all high-lying cranial nerves [4, 5], and it does not leave a long and visible scar with an increased risk of permanently altered skin sensation $[3,34]$. Shorter balloon catheters (in case of the decision on endovascular rather than surgical CCA clamping) along shorter balloon/ stent delivery systems would be welcomed by operators using the transcervical access and it might facilitate further penetration of the strategy. While the current (markedly excessive for the trans-carotid route, cf. Figure 1) length of the catheters (including flow-reversal catheters) makes their use for transcervical CAS rather inconvenient (cf. Figure 1), it is our experience that the catheter use (in comparison to a direct through-the-access-sheath procedure), appears to be associated with an increased access system stability. Customization (significant shortening) of the balloon catheters (for those who would prefer to take advantage of an increased system stability and would refer to perform endovascular rather than surgical CCA clamp) would be an advantage, similar to customization of the wires, and the balloon and stent delivery systems. The flow reversal circuit tubing should be no longer than needed to reduce resistance to flow. An important point on the operator skills and attention part is a high-level care that needs to be taken to ensure the lesion 'notouch' prior to establising flow reversal.

One fundamental contraindication to the use of the transcervical access in CAS is any significant athero-occlusive disease at the anticipated level of CA cannulation, another is short neck with a low CCA division [3, 5]. Other contraindications are any anatomic abnormalities overlying CA at the base of the neck. The disadvantage of open CCA cutdown offers, at the same time, the advantage (over any percutaneous approach with the current techniques and devices) of a fully controlled vessel cannulation in the critical anatomic region and of a fully controlled artery access site closure. There is currently no other CCA closure that would be more controllable and effective than surgical closure.

The role of ECA clamping to prevent potential ECAICA flow during CCA clamping remains a highly debated and unresolved issue [3-5, 23, 24]. It is highly likely, however, that a robust flow reversal may make the role of ECA exclusion negligible. Although larger data series are needed, we have not excluded ECA in 3/4 of cases and there was absence of cerebral embolism despite the embolic material generated with lesion crossing and predilatation (Figure $2 \mathrm{~B}$ ). Ensuring flow reversal robustness points to the role of the sheath/catheter and tubing diameter that, if relatively small, may be corrected with syringe aspiration(s) (whose feasibility should always be ensured in carotid flow reversal) while it appears to have been rightly optimized in the EnRoute TCAR kit.

In addition to a high-level procedural safety, TCAR minimizes cranial nerve injury associated with carotid surgery $[10,11]$ that is one of the important drawbacks of CEA from the patient's perspective [34]. Overall evidence, inclusive of a significant proportion ( $\approx 30-60 \%)$ of 30 -day strokes occurring with $1^{\text {st }}$ generation carotid stents post-procedurally in relation to on-going post-procedural plaque embolism [27] (see CREST, CAPTURE, ICSS and other data reviewed recently in Ref. 8 and Ref. 12) is consistent with (not unexpected) TCAR first-generation opencell stent inability to eliminate post-procedural strokes $[10,11,26]$. Conversely, the MicroNET-covered stent design shows an effective minimization of post-procedural embolism [29] (that is a measure of the clinical stroke risk [35]) and elimination of post-procedural strokes [15-18, $30,31]$, suggesting that the transcervical flow reversal approach to CAS will need to incorporate advances in the carotid stent design that translate into improved periand post-procedural neurologic outcomes.

Investigator-initiated TOP-GUARD Study (Carotid Artery Revascularization Using Iranscarotid flow Reversal Cerebral Protection And CGUARD MicroNET Embolic Prevention Stent System To Reduce Strokes; NCT04547387) is effectively continuing recruitment. Another study focused on post-procedural evaluation of the endovascular carotid artery plaque sequestration and lumen reconstruction using intravascular ultrasound (CGuard OPTIMA: OPTIMAl endovascular exclusion of high-risk carotid plaque using the CGuard ${ }^{\mathrm{TM}}$ MicroNET-covered embolic prevention stent system in consecutive patients with symptoms or signs of carotid stenosis-related brain injury: An intravascular ultrasound-controlled investigator-initiated multicentric multi-specialty study; NCT04234854) is close to completing recruitment.

\section{Conclusions}

In this technical note expanded with clinical and imaging data in a series of very high-risk patients, we report our recent experience in transcervical carotid ar- 
tery revascularization in neurologically symptomatic patients. We show how using routinely available vascular surgery and endovascular angiology equipment and tools for transient flow reversal to protect the brain from intra-procedural embolism, paired with the use of MicroNET-covered embolic prevention stents to exclude the symptomatic plaque from the lumen, enable an effective restoration of normal anatomy with prevention of plaque-related embolism. Use of a plaque-insulating stent system overcomes limitations of $1^{\text {st }}$ generation carotid stents, increasing the spectrum of carotid lesions routinely suitable for endovascular stent-assisted lumen reconstruction to include highly-emboligenic lesions for which CEA has been a historic first choice. As it is well known today that optimized medical therapy alone is not sufficient to effectively prevent strokes in relation to atherosclerotic carotid stenosis, minimally-invasive and clinically-effective peri-procedural and post-procedural prevention of post-procedural cerebral embolism (and thus post-procedural stroke risk prevention), taken together with improved stroke risk stratification at the lesion and patient level, may soon transform the field of stroke prevention in relation to carotid artery stenosis. It is clear today that the transcervical flow reversal approach to CAS [3-5, 9-11, 29, 36], will need to incorporate advances in the carotid stent design that, as accumulating evidence shows, translate into improved peri- and post-procedural cerebral outcomes.

\section{Acknowledgments}

We would like to acknowledge contributions from Ewa Węglarz RN MSc, Łukasz Tekieli MD PhD, and Adam Mazurek MD PhD. We are grateful for support from the John Paul II Hospital in Krakow Hybrid Room staff (Heads: Zbigniew Woszczyna MSc, Ewa Żądło RN MSc), our colleagues from the Anesthesia Teams (Head: Robert Musiat MD PhD), our diagnostic radiology team (Dr. Małgorzata Urbańczyk and Paweł Banyś MSc) and consulting stroke neurologists. We thank Paweł Kołacz MSc for his editorial assisstance.

This work was supported by the science fund of the John Paul II Hospital, Krakow, Poland (no. FN11/2020 to $P M)$.

\section{Conflict of interest}

MT: Proctoring/Consulting: Medtronic, Terumo. PM: Proctoring/Consulting: Abbott, InspireMD, Medtronic. PM serves on the European Society of Cardiology (ESC) Stroke Council Scientific Documents Task Force.

In 2018 the John Paul II Hospital in Krakow was certified as an international 'TCAR Center of Excellence'. There have been no financial relationships (or other relationships that might be perceived as creating a conflict of interest) of the authors with the TCAR EnRoute system manufacturer
We are grateful to SilkRoad Medical (USA) for their unrestricted provision (until the COVID-19 pandemic) of their CE-marked TCAR systems (EnRoute) for our routine clinical use.

\section{References}

1. Musialek P, Hopf-Jensen S. Carotid artery revascularization for stroke prevention: a new era. J Endovasc Ther 2017; 24: 138-48.

2. Grunwald IQ, Reith W, Kühn AL, et al. Proximal protection with the Gore PAES can reduce DWI lesion size in high-grade stenosis during carotid stenting. Eurolntervention 2014; 10: 271-6.

3. Chang DW, Schubart PJ, Veith FJ, Zarins CK. A new approach to carotid angioplasty and stenting with transcervical occlusion and protective shunting: why it may be a better carotid artery intervention. J Vasc Surg 2004; 39: 994-1002.

4. Criado E, Doblas M, Fontcuberta J, et al. Transcervical carotid artery angioplasty and stenting with carotid flow reversal: surgical technique. Ann Vasc Surg 2004; 18: 257-61.

5. Pipinos II, Johanning JM, Pham CN, et al. Transcervical approach with protective flow reversal for carotid angioplasty and stenting. J Endovasc Ther 2005; 12: 446-53.

6. Pieniazek P, Musialek P, Dzierwa K, et al. Flow reversal for proximal neuroprotection during endovascular management of critical symptomatic carotid artery stenosis coexisting with ipsilateral external carotid artery occlusion. J Endovasc Ther 2009; 16: 744-51.

7. Wissgott C, Schmidt W, Brandt-Wunderlich C, et al. Clinical results and mechanical properties of the CGuard double-layered embolic protection stent. J Endovasc Ther 2017; 24: 130-7.

8. Musiałek P, Roubin GS. Double-layer carotid stents: from the clinical need, through a stent-in-stent strategy, to effective plaque isolation... the Journey Toward Safe Carotid Revascularization using the endovascular route. J Endovasc Ther 2019; 26: 572-7.

9. Leal I, Orgaz A, Flores Á, et al. A diffusion-weighted magnetic resonance imaging-based study of transcervical carotid stenting with flow reversal versus transfemoral filter protection. J Vasc Surg 2012; 56: 1585-90.

10. Kashyap VS, Schneider PA, Foteh M, et al. Early outcomes in the ROADSTER 2 study of transcarotid artery revascularization in patients with significant carotid artery disease. Stroke 2020; 51: 2620-9.

11. Liang P, O'Donnell TFX, Cronenwett JL, et al. Vascular quality initiative risk score for 30-day stroke or death following transcarotid artery revascularization. J Vasc Surg 2020; S07415214(20)32279-5 (epub ahead of print).

12. Musiałek P, Hopkins LN, Siddiqui AH. One swallow does not a summer make but many swallows do: accumulating clinical evidence for nearly-eliminated peri-procedural and 30-day complications with mesh-covered stents transforms the carotid revascularisation field. Adv Interv Cardiol 2017; 13: 95-106.

13. Nikas D, Reith W, Schmidt A, et al. Prospective, multicenter European study of the GORE flow reversal system for providing neuroprotection during carotid artery stenting. Catheter Cardiovasc Interv 2012; 80: 1060-8.

14. Pieniazek P, Musialek P, Kablak-Ziembicka A, et al. Carotid artery stenting with patient- and lesion-tailored selection of the neuroprotection system and stent type: early and 5 -year results from a prospective academic registry of 535 consecutive procedures (TARGET-CAS). J Endovasc Ther 2008; 15: 249-62. 
15. Musialek P, Mazurek A, Trystula M, et al. Novel PARADIGM in carotid revascularisation: prospective evaluation of All-comer peRcutaneous cArotiD revascularisation in symptomatic and Increased-risk asymptomatic carotid artery stenosis using CGuard $^{\mathrm{TM}}$ MicroNet-covered embolic prevention stent system. Eurolntervention 2016; 12: e658-70.

16. Schofer J, Musiałek P, Bijuklic K, et al. A prospective, multicenter study of a novel mesh-covered carotid stent: the CGuard CARENET Trial (Carotid Embolic Protection Using MicroNET). JACC Cardiovasc Interv 2015; 8: 1229-34.

17. Mazurek A, Partyka L, Trystula M, et al. Highly-calcific carotid lesions endovascular management in symptomatic and increased-stroke-risk asymptomatic patients using the CGuard ${ }^{\text {TM }}$ dual-layer carotid stent system: analysis from the PARADIGM study. Catheter Cardiovasc Interv 2019; 94: 149-56.

18. Mazurek A, Boratynska A, Malinowski KP, et al. MicroNET-covered stents for embolic prevention in patients undergoing carotid revascularisation: twelve-month outcomes from the PARADIGM study. Eurolntervention 2020; 16: e950-2.

19. Mazurek A, Bugurov S, Musialek P. Short-term double layer mesh stent patency for emergent or elective carotid artery stenting: a single center experience. Stroke 2019; 50: e433-4.

20. Tekieli Ł, Musiałek P, Kabłak-Ziembicka A, et al. Severe, recurrent in-stent carotid restenosis: endovascular approach, risk factors. Results from a prospective academic registry of 2637 consecutive carotid artery stenting procedures (TARGET-CAS). Adv Interv Cardiol 2019; 15: 465-71.

21. Musialek P, Stabile E. Residual plaque prolapse with novel dual-layer carotid stents: is it mesh-covered or not? Eurolntervention 2017; 13: 1266-8.

22. Badacz R, Przewłocki T, Karch I, et al. Low prevalence of collateral cerebral circulation in the circle of Willis in patients with severe carotid artery stenosis and recent ischemic stroke. Adv Interv Cardiol 2015; 11: 312-7.

23. Ohki T, Parodi J, Veith FJ, et al. Efficacy of a proximal occlusion catheter with reversal of flow in the prevention of embolic events during carotid artery stenting: an experimental analysis. J Vasc Surg 2001; 33: 504-9.

24. Ouriel K, Greenberg RK, Sarac TP. Hemodynamic conditions at the carotid bifurcation during protective common carotid occlusion. J Vasc Surg 2001; 34: 577-80.

25. Badacz R, Kabłak-Ziembicka A, Urbańczyk-Zawadzka M, et al. Magnetic resonance imaging and clinical outcome in patients with symptomatic carotid artery stenosis after carotid artery revascularization. Adv Interv Cardiol 2017; 13: 225-32.

26. Criado E, Fontcuberta J, Orgaz A, et al. Transcervical carotid stenting with carotid artery flow reversal: 3-year follow-up of 103 stents. J Vasc Surg. 2007; 46: 864-9.

27. Schofer J, Arendt M, Tübler T, et al. Late cerebral embolization after emboli-protected carotid artery stenting assessed by sequential diffusion-weighted magnetic resonance imaging. JACC Cardiovasc Interv 2008; 1: 571-7.

28. Kotsugi M, Takayama K, Myouchin K, et al. Carotid artery stenting: investigation of plaque protrusion incidence and prognosis. JACC Cardiovasc Interv 2017; 10: 824-31.

29. Karpenko A, Ignatenko P, Bugurov S. Randomized controlled trial of conventional versus Micronet-covered stent use in percutaneous neuroprotected carotid artery revascularization: peri-procedural and 30-day diffusion-weighted magnetic resonance imaging and clinical outcomes. https://media.pcronline.com/ diapos/PCReCourse2020/300-20200625_1610_Hotline_and_In-
novation_Channel_Ignatenko_Pavel_0000_(645)/MainRecord. mp4 EuroPCR 2020 Late-Breaking Clinical Trial. (accessed 30 November 2020).

30. Stabile E, de Donato G, Musialek P, et al. Use of dual-layered stents in endovascular treatment of extracranial stenosis of the internal carotid artery: results of a patient-based meta-analysis of 4 clinical studies. JACC Cardiovasc Interv 2018; 11: 2405-11.

31. Stabile E, de Donato G, Musialek P, et al. Use of dual-layered stents for carotid artery angioplasty: 1-year results of a patient-based meta-analysis. JACC Cardiovasc Interv 2020; 13 : 1709-15.

32. Musialek P, Dabrowski W, Mazurek A, et al. Quantitative virtual histology for in vivo evaluation of human atherosclerosis a plaque biomechanics-based novel image analysis algorithm: validation and applications to atherosclerosis research. In: Balocco S (ed.). Intravascular Ultrasound: From Acquisition to Advanced Quantitative Analysis. Elsevier 2000.

33. Piątek J, Kędziora A, Dzierwa K, et al. Hybrid one-day coronary artery bypass grafting and carotid artery stenting - cardiac surgeons' perspective on the procedure's safety. Adv Interv Cardiol 2018; 14: 99-102.

34. Trystuła M, Tomaszewski T, Pąchalska M. Health-related quality of life in ischaemic stroke survivors after carotid endarterectomy (CEA) and carotid artery stenting (CAS): confounder-controlled analysis. Adv Interv Cardiol 2019; 15: 226-33.

35. Traenka C, Engelter ST, Brown MM, et al. Silent brain infarcts on diffusion-weighted imaging after carotid revascularisation: a surrogate outcome measure for procedural stroke? A systematic review and meta-analysis. Eur Stroke J 2019; 4: 127-43.

36. Wang SK, Severance S, Westin GG, et al. Perioperative and 1-year transcarotid revascularization outcomes in symptomatic patients. J Vasc Surg 2020; 72: 2047-53. 